\title{
Enfermedad de injerto contra huésped, una presentación inusual
}

\author{
Luz Adriana Vásquez ${ }^{1}$, Andrés $\mathrm{Mazo}^{2}$, Ana Cristina Ruiz ${ }^{3}$ \\ 1. Médica dermatóloga, Hospital Pablo Tobón Uribe, Medellín, Colombia \\ 2. Médico interno, Universidad Pontificia Bolivariana, Medellín, Colombia \\ 3. Médica dermatopatóloga, Hospital Pablo Tobón Uribe, Medellín, Colombia.
}

\section{RESUMEN}

La enfermedad aguda de injerto contra huésped es una complicación común de los trasplantes alogénicos, con impacto en la morbimortalidad. Usualmente, las manifestaciones dermatológicas junto con las gastrointestinales son las más frecuentes y la presentación clásica es el cuadro clínico por afección de estos dos sistemas; sin embargo, las primeras suelen ser las de más rápida aparición, lo cual es importante para hacer un diagnóstico oportuno. Entre las dermatológicas, el brote máculo-papular es la manifestación más frecuente, aunque se han descrito otras como las placas liquenoides o alopécicas o, simplemente, la dishidrosis.

Se presenta el caso de un paciente con máculas hiperpigmentadas en la cara, una forma clínica atípica de esta enfermedad.

PALABRAS CLAVE: enfermedad de injerto contra huésped; trasplante.

\section{SUMMARY}

The acute graft-versus-host disease is a common complication of allogeneic transplants with impact in morbidity and mortality. The dermatological and gastrointestinal manifestations are usually the most frequent and classical presentation; however, the dermatological signs are usually the earliest which is important for a timely diagnosis; the maculopapular rash is the most common dermatological manifestation. They have been described in the literature as lichenoid or alopecic plaques or just dyshidrosis.

A case of hyperpigmented macules on the face is presented and it represents an atypical clinical form of this disease.

KEY WORDS: Graft-versus-host, transplants.

\section{INTRODUCCIÓN}

Se trata de un paciente de sexo masculino de 60 años de edad, con antecedentes de diabetes mellitus de tipo 2 y de linfoma difuso de células $\mathrm{B}$, para el cual recibió trasplante alogénico de intensidad reducida. La histocompatibilidad del donante se estableció mediante HLA de primera línea.

Antes del trasplante, había iniciado tratamiento con ciclosporina a la cual presentó 

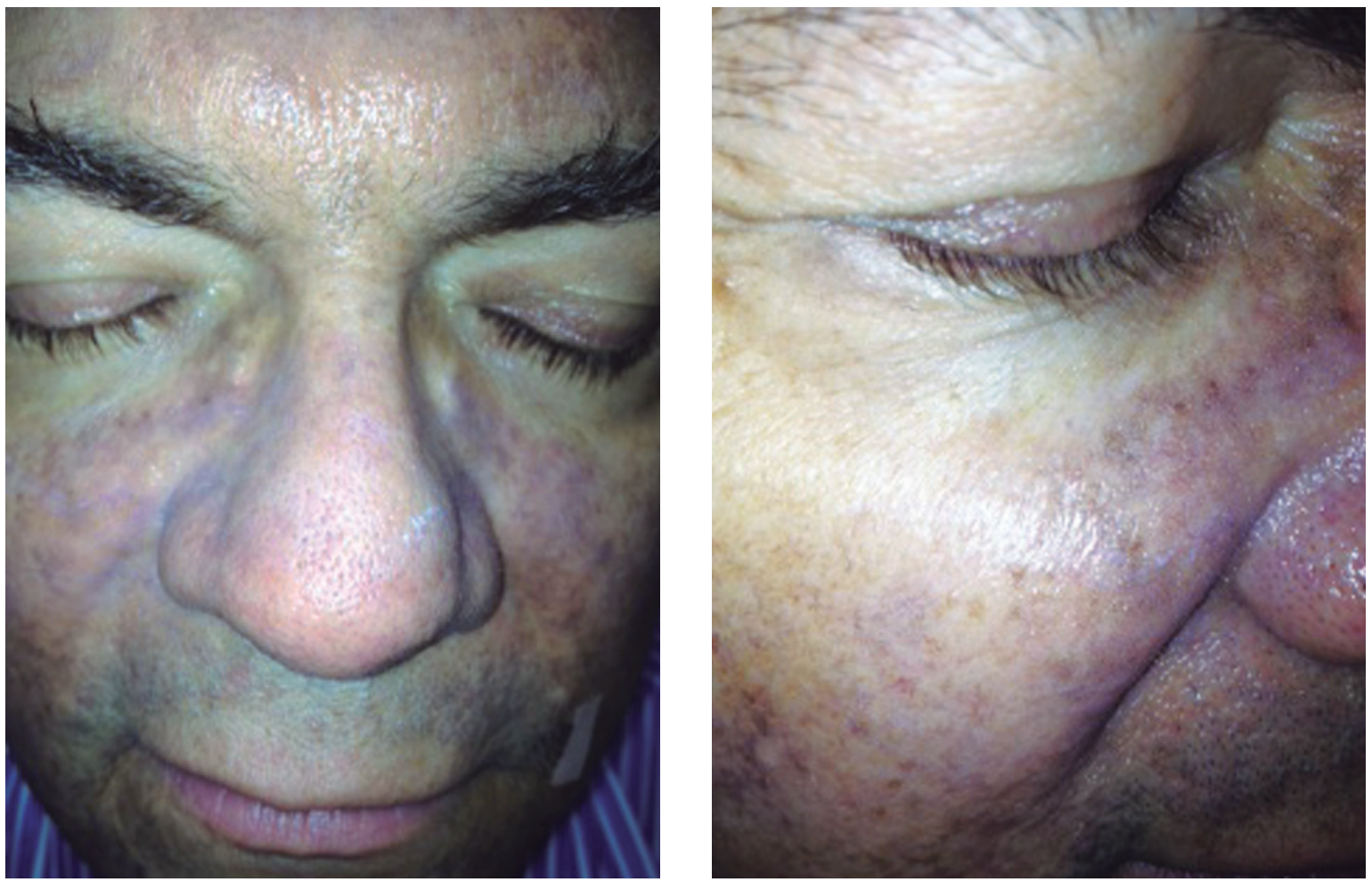

FIGURA 1 Y 2. Foto Paciente con maculas café y placas eritemato violáceas distribuidas en mejillas con bordes irregulares simétricas .

intolerancia, por lo cual se inició un esquema con $1 \mathrm{~g}$ diario de micofenolato de mofetilo durante ocho semanas. Después del trasplante, se continuó el tratamiento con un gramo diario de micofenolato de mofetilo (con un tiempo programado de seis meses), aciclovir, fluconazol, metformina y gabapentín. Ultima quimioterapia recibida 8 meses antes del trasplanté.

Cuatro meses después del trasplante (día 119), consultó por un cuadro clínico de 15 días de evolución consistente en deposiciones diarreicas y lesiones eritemato-violáceas asintomáticas localizadas en las mejillas.

En el examen físico se observaron máculas de color café y placas eritemato-violáceas (figura 1), algunas de ellas con descamación, en las mejillas, los surcos nasogenianos y la región interciliar. Las mucosas estaban semisecas debido a la deshidratación por la diarrea. No había adenopatías ni hepatomegalia.

En los exámenes de laboratorio, se encontró: 1.100 leucocitos por $\mu \mathrm{l}, 41 \%$ de linfocitos, $11,8 \mathrm{~g} / \mathrm{dl}$ de hemoglobina y 149.000 plaquetas por mm3; la pruebas de función hepática fueron normales.

En el estudio de histopatología de la biopsia de piel, se informó: leve acantosis, ortoqueratosis compacta y queratinocitos en la capa basal (figura 2); se interpretó como concluyente para enfermedad aguda de injerto contra huésped, de grado II.

Recibió tratamiento con glucocorticoides sistémicos por vía oral: $12 \mathrm{mg}$ diarios de flazacort durante cinco días $\mathrm{y}$, después, $6 \mathrm{mg}$ diarios durante 10 días. El tratamiento tópico consistió en la aplicación de aceponato de metilprednisolona en crema dos veces al día durante seis días. Se obtuvo resolución de las lesiones. El esquema de inmunosupresores no se cambió.

\section{DISCUSIÓN}

La enfermedad de injerto contra huésped es una reacción inmunitaria de las células del donante contra los tejidos del huésped que ocurre, generalmente, después de trasplantes hematopoyéticos alogénicos ${ }^{(1)}$.

Los sistemas más afectados son la piel (81\%), el gastrointestinal $(54 \%)$ y el hígado $(50 \%){ }^{(2)}$; y la presentación clínica clásica comprende la sintomatología de estos dos primeros sistemas, como en el caso presentado.

Los criterios del 2005 de los National Institutes of Health (NIH) permiten diferenciar entre la variante aguda y la 
crónica, y hacen énfasis en que el diagnóstico depende de los hallazgos clínicos más que del tiempo de evolución (3). Esta complicación ocurre en 20 a $65 \%$ de los casos y es responsable del $50 \%$ de las muertes no atribuibles a la recidiva tumoral ${ }^{(4)}$.

Clásicamente, se ha considerado que la forma aguda ocurre en las primeras seis semanas después del trasplante y, la crónica, a partir de los 100 días. Sin embargo, además de las nuevas recomendaciones de los NIH, se agregaron otras categorías, entre las cuales se incluyen la forma aguda de comienzo tardío y el síndrome de superposición como variante de la forma crónica. No obstante, aún no está claro si estos dos nuevos grupos implican diferencias en la mortalidad o en otros resultados importantes ${ }^{(5)}$.

El principal factor de riesgo es la disparidad del HLA, la cual ha sido ampliamente estudiada. La incidencia de la enfermedad aguda entre hermanos con HLA idéntico es de alrededor del $20 \%$, en donantes no relacionados con HLA idéntico, de $30 \%$, y en casos de disparidad en uno de los dos alelos, de $40 \%{ }^{(2)}$. Otros factores de riesgo son la diferencia del sexo entre donantes, específicamente en casos de donante femenino con recipiente masculino(5), y la intensidad del esquema inmunosupresor posterior al trasplante. En este paciente, se trataba de un donante sin relación de parentesco, con HLA concor-

\section{"Las manifestaciones clínicas más comunes de la forma aguda son un exantema maculopapular que suele iniciarse en las palmas y las plantas, pero que puede afectar cualquier parte del cuerpo; en las formas más graves, se observa una reacción similar a la necrosis epidérmica toxica y debe tenerse en cuenta en los diagnósticos diferenciales"}

dante y se desconocía el sexo del donante; asimismo, la tamización para citomegalovirus había sido negativa.

Las manifestaciones clínicas más comunes de la forma aguda son un exantema maculopapular que suele iniciarse en las palmas y las plantas, pero que puede afectar cualquier parte del cuerpo; en las formas más graves, se observa una reacción similar a la necrosis epidérmica toxica y debe tenerse en cuenta en los diagnósticos diferenciales ${ }^{(6)}$.

Dicho exantema tiene predilección por las áreas distales (acral) y presenta un aspecto hemorrágico en los pacientes con trombocitopenia, en quienes su pico de aparición es a las seis semanas después del trasplante ${ }^{(7)}$. La forma crónica se presenta en $40 \%$ de los casos y se caracteriza por la ausencia de las manifestaciones clínicas clásicas de la forma aguda. La enfermedad crónica puede clasificarse como progresiva o de novo. La primera se caracteriza por evolucionar desde una fase aguda, estática, en la cual hay un periodo libre de enfermedad; en la segunda, no existe el antecedente de enfermedad aguda previa. La forma progresiva es de peor pronóstico. Las manifestaciones cutáneas de la forma crónica, que permiten hacer el diagnóstico en el contexto adecuado, son poiquilodermia, queratosis parecida al liquen plano, o lesiones escleróticas o morfeiformes ${ }^{(5)}$.

En la literatura científica se han reportado presentaciones atípicas de esta enfermedad, tanto en la forma aguda como en la crónica ${ }^{(8)}$. Se han descrito manifestaciones como hiperpigmentación, lesiones psoriasiformes ${ }^{(9,10)}$ y placas liquenoides foliculares ${ }^{(1)} \mathrm{o}$ del tipo de la dermatitis de contacto ${ }^{(12)}$.

En el presente caso, la afectación de la piel y del sistema gastrointestinal (diarrea hipersecretora) es clásica de la enfermedad; sin embargo, es atípica la afección cutánea, por la hiperpigmentación de las mejillas y la nariz. En el caso de un paciente con trasplante, debe sospecharse la enfermedad de injerto contra huésped ante cualquier lesión en la piel, la cual puede ser su primera manifestación.

Entre los medicamentos de primera línea de tratamiento están los corticoides y los inhibidores de la calcineurina. En cuanto a los corticoides, la vía tópica es de elección para las manifestaciones cutáneas. Su potencia y tiempo de administración dependen del área corporal afectada y el grado de la lesión, teniendo siempre en cuenta que se deben evitar los esteroides muy potentes en la cara. El valerato de betametasona puede usarse dos veces al día durante 4 a 12 semanas. Si se disminuye la potencia del corticoide (por ejemplo, en esquemas con butirato de clobetazol), se recomienda aumentar el tiempo de tratamiento hasta los 12 meses. En las palmas y en las 


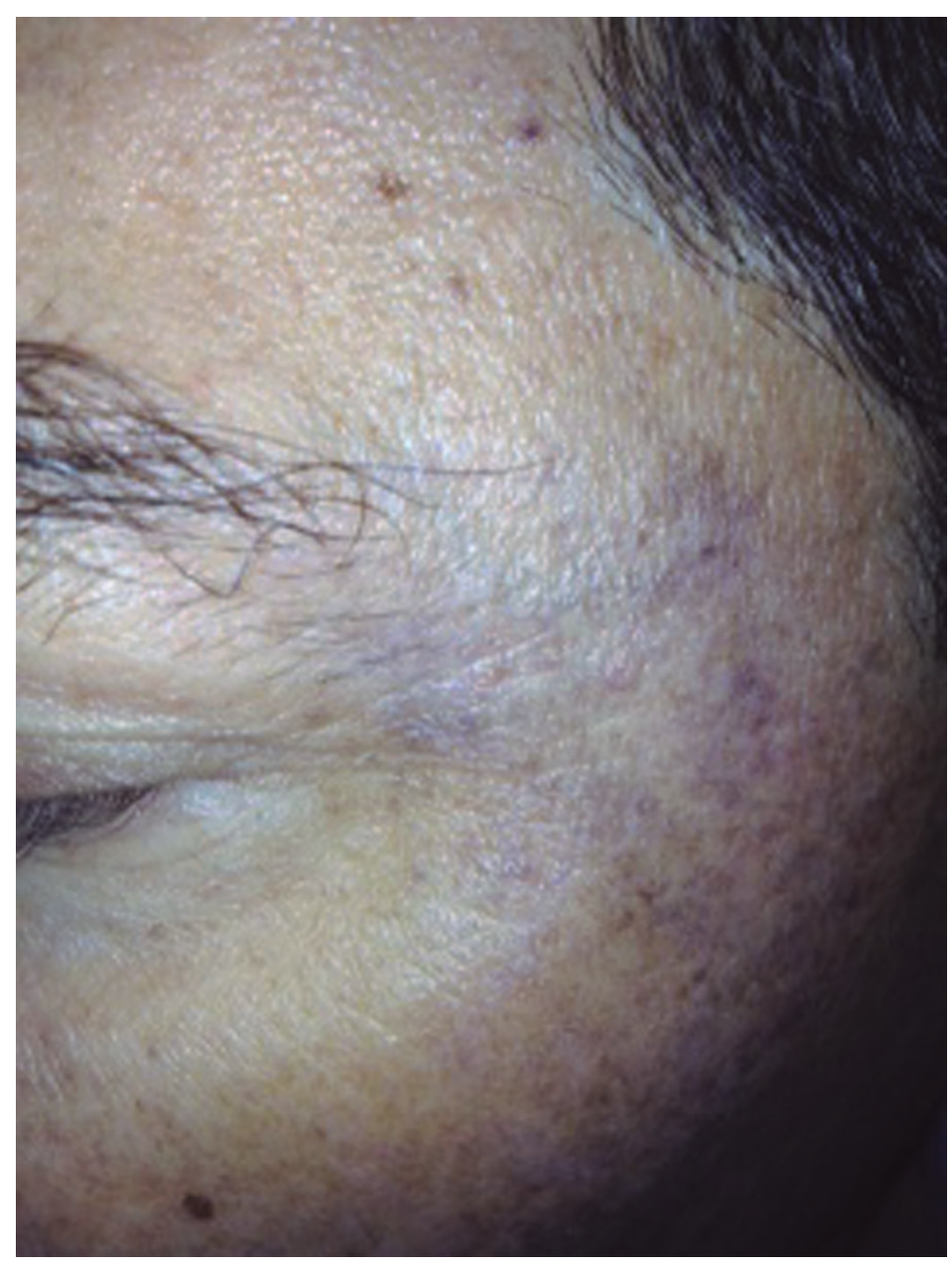

FIGURA 3. Paciente con placas eritemato violaceas paraciliares.

plantas, se recomienda el propionato de clobetazol durante 4 a 12 semanas. En menores de 15 años, se recomienda el tracolimus. En el tratamiento sistémico puede usarse la prednisolona, $1 \mathrm{mg} / \mathrm{kg}$ durante 14 días, con la cual se han logrado tasas de remisión completa en $35 \%$ de los casos; y, para la enfermedad de grado III a IV, se puede escalar la terapia a $2 \mathrm{mg} / \mathrm{kg}$ de metilprednisolona por vía intravenosa.

Cuando falla el tratamiento con esteroides, específicamente con metilprednisolona después de cinco días, se recomiendan los de segunda línea, entre los cuales se encuentran: la fotoforesis extracorpórea, los anticuerpos anti-TNF, la rapamicina y el micofenolato. Entre los de tercera línea, se encuentran el uso de células madres mesenquimales (en estudio) y medicamentos como el alemtuzumab.

\section{REFERENCIAS}

1. Jacobsohn DA, Vogelsang GB. Acute graft versus host disease. Orphanet J Rare Dis. 2007;2:35.

2. Montoya C, Sierra M, Vidal A. Enfermedad injerto contra huésped. Rev Colomb Dermatol. 2016;24:90-100.

3. Vigorito AC, Campregher PV, Storer BE, Carpenter PA, Moravec CK, Kiem H-P, et al. Evaluation of NIH consensus criteria for classification of late acute and chronic GVHD. Blood. 2009;114:702-8.

4. Lowsky R, Takahashi T, Liu YP, Dejbakhsh-Jones S, Grumet FC, Shizuru JA, et al. Protective conditioning for acute graftversus-host disease. N Engl J Med. 2005;353:1321-31.

5. Filipovich AH, Weisdorf D, Pavletic S, Socie G, Wingard JR, Lee SJ, et al. National Institutes of Health consensus development project on criteria for clinical trials in chronic graft- 
versus-host disease: I. Diagnosis and staging working group report. Biol Blood Marrow Transplant. 2005;11:945-56.

6. Dignan FL, Clark A, Amrolia P, Cornish J, Jackson G, Mahendra $\mathrm{P}$, et al. Diagnosis and management of acute graftversus-host disease. Br J Haematol. 2012;158:30-45.

7. Gale RP, Bortin MM, van Bekkum DW, Biggs JC, Dicke KA, Gluckman E, et al. Risk factors for acute graft-versus-host disease. Br J Haematol. 1987;67:397-406.

8. Cornejo CM, Kim EJ, Rosenbach M, Micheletti RG. Atypical manifestations of graft-versus-host disease. J Am Acad Dermatol. 2015;72:690-5.

9. Taguchi S, Kawachi Y, Fujisawa Y, Nakamura Y, Furuta J, Otsuka F. Psoriasiform eruption associated with graft-versushost disease. Cutis. 2013;92:151-3.

10. Matsushita T, Hasegawa M, Shirasaki F,Fujimoto M, Yamazaki H, Sato S, et al. A case of acute cutaneous graft-versushost disease mimicking psoriasis vulgaris. Dermatology. 2008;216:64-7.

11. Goiriz R, Delgado-Jiménez Y, Fernández-Peñas P, Fraga J, García-Diez A, Fernández-Herrera J. Atypical early follicular graft-vs-host disease. Arch Dermatol. 2006;142:1237-8.

12. Kuskonmaz B, Güçer S, Boztepe G, Cetin M, Uckan D. Atypical skin graft-vs.-host disease following bone marrow transplantation in an infant. Pediatr Transplant. 2007;11:214-6. 